\title{
On the Ethical Issues of Gene-enhancement in Different Stages of Human Development
}

\author{
Jingtao Jiang \\ Research Centre of Medical Humanities, Zunyi Medical University, Zunyi, China
}

Email address:

jjt@zmu.edu.cn

\section{To cite this article:}

Jingtao Jiang. On the Ethical Issues of Gene-enhancement in Different Stages of Human Development. Advances in Sciences and Humanities. Vol. 7, No. 2, 2021, pp. 19-24. doi: 10.11648/j.ash.20210702.12

Received: April 14, 2021; Accepted: April 28, 2021; Published: May 14, 2021

\begin{abstract}
The ethics of human enhancement technology are highly controversial. The purpose of this paper is to provide an ethical justification for the genetic enhancement technology and then extend it to the ethical feasibility of other human enhancement technologies. According to the customs of most countries in the world, the age of 18 is generally regarded as the threshold of adulthood. Non-adults under 18 can be divided into the embryonic period from the fertilized egg to the eighth week and the growing period from the ninth week to the age of 18 . Genetic enhancement in embryonic period has been criticized by a series of ethical charges with "germline change" as the core. Although genetic enhancement in the growing period avoids the critical opinion of "germline change", it is still criticized by a series of ethical charges with "human-nature change" as the core. Genetic enhancement in the adulthood will successfully avoid the two fatal charges of "germline change" and "human-nature change", while responding well to other ethical charges. The conclusion of this paper is that adult gene enhancement is ethically feasible, and further application of other adult human enhancement techniques is also ethically feasible.
\end{abstract}

Keywords: Genetic Enhancement, Embryonic Period, Growing Period, Adulthood

\section{Introduction}

As for the various stages of human life, although cultural differences vary from country to country, there is still some basic consensus. On these basic consensus, and in accordance with the needs of argument in this paper, we hereby divide the human procedure from fertilized egg to death into three major stages. The first stage is the embryonic period from the union of sperm and egg to the end of the eighth week, the second stage starts from the ninth week until the age of 18 , and the third stage is the adult period from the age of 18 until the end of death. Embryo stage, especially before day 14, does not have the basic human characteristics and is often treated as an object, so many people can accept gene editing, induced abortion, or even destroy the surplus frozen embryos of test-tube babies. [1]Fetuses, infants, children, and adolescents in their growing period possess human potential and some human characteristics, and so are often treated as human beings. Although they do not enjoy full civil rights, they are treated as human beings in the legal sense and will be given additional preferential treatment. Hurting a growing person is worse than hurting an adult. The adult individual enjoys full civil rights and duties, enjoys all kinds of ethical and legal protections prescribed by human standards, and is also fully responsible for his own choices and actions.

On the basis of such division, this paper firstly analyzes the ethical issues of gene enhancement in human embryo, then the ethical issues of gene enhancement in growing stage, and finally the ethical issues of gene enhancement in adulthood. Based on the above analysis, this paper concludes that the genetic enhancement of adults is the free choice and self-risk based on the maintenance of human species and human nature, and so is acceptable. This argument can be extended to other ethical issues of human enhancement technology.

\section{Ethical Problems on Gene-enhancement During Human Embryonic Period}

Genetic enhancement in Embryonic period refers to the modification of DNA (deoxyribonucleic acid) in the nucleus of a human embryo, sperm, or egg cell by gene editing technology to add some beneficial properties that are not 
inherently embodied. Because the genetic enhancement of human embryo challenges a series of traditional ideas and norms, it has aroused a lot of controversies. At present, the public voice towards human embryo gene enhancement is mainly criticism and denial. The power of supporters is very weak, which often leads to a series of criticism and opposition immediately after appearing, such as the case of He Jiankui's gene-edited baby. [2]

On the side of supporters, Paul Knoepfler is sympathetic to the idea of genetic enhancement in human embryos, arguing that society cannot prevent all scientists from using gene editing to create "designer babies". [3] The underlying reason for this is that it does have some benefits for humans: (a) It is conducive to the development of scientific research. Human embryonic gene editing is now a powerful tool for scientific research, which can improve our understanding of early embryonic development, our exploring of genetic diseases, and help us unlock the secrets of human development and disease origin. [4] (b) it is conducive to ameliorate human defects. Human embryo gene enhancement can prevent gene diseases, overcome the inherent defects, and make human become more adaptable to the changes of social and natural environment. [5] (c) it is conducive to the competition of our offspring. By genetically enhancing our babies, we can make our own offspring smarter, stronger and faster, giving them an edge over their peers.

On the side of opponents, the main argument is that genetic enhancement in human embryos will altered the human germline, creating new species that are inhuman and threatening the survival and development of the original human species. [6] (a) Human embryo gene enhancement is the modification of human germ cells to create other artificial creations different from human cells, and then gradually replace the original human body cells in the further development. At the cellular level, they are different from human. They are no longer human and are some new species. Even they share a large number of genes with humans in general, the signature event of germ-cell alterations has led us to define them as a new species, similar to the gorilla. (b) As for babies who are naturally born with mutations, their incidence is extremely low. Among them, those that are not conducive to survival will be eliminated by natural selection, while those that are conducive to survival will take an extremely long time to mate and reproduce with other normal humans, and will be eventually integrated into the gene pool and civilization system of human beings. While the artificially gene-enhancement new species can be mass-produced to form an interest alliance, which will not integrate into the original human community but will compete and conflict with them for resources. They are highly similar to human beings and at the same time surpass human beings, which will inevitably pose a survival challenge and even extinction crisis to us. (c) In addition, babies born with genetic mutations in nature are unintentional incidental products, which are inevitable byproducts of mankind itself as a whole. Since it is the common business of the whole human race, and should be borne by the whole human race for its advantages and disadvantages. While gene-edited babies, on the other hand, can be consciously mass-produced. They are the product of the selfish interests or prejudices of a few scientists or politicians, but the consequences of their benefits and disadvantages would be borne by the whole of humanity, which is obviously unfair.

On the side of opponents, there are some other ethical criticisms from physiological, psychological, and social perspectives. In the physiological aspect: (a) The lack of adequate security guarantees. Human embryos are not edited accurately enough and will deviate from their intended targets. Such off-target mutations can lead to cancer and other pathological development. [7] (b) The line between treatment and enhancement is blurred. Treatment is the correction of physiological indicators to return them to the normal range, while enhancement is the raising of some indicators to elevate them to beyond the normal human range, but this normal human range is a very vague definition. Treatment of the former belongs to the traditional medicine and is not controversial, but enhancement of the latter will go beyond the realm of traditional medicine and is going to trigger a series of huge, unforeseen impacts. Genetic enhancement must be prohibited until the boundaries between treatment and enhancement are clearly defined and the uncertain impacts of enhancement are addressed. [8] In the psychological aspect: (a) The change of traditional human nature. Human beings, based on the natural probability of genes, form a series of inherent human characteristics, while gene editing technology will intentionally manipulate human gene to affect and change original human characteristics. For example, it will expand the boundless aspirations of human beings, strengthen anthropocentrism, challenge traditional values of humility, responsibility and solidarity, and so on. [9] (b) The challenge of human dignity. Gene-edited individuals are often regarded as a kind of material or goods, whose life and even destiny will be controlled by someone that behind the gene editing surgery. At the same time, people who have been genetically edited will be greatly disturbed by the disclosure of their genetic privacy. [10] (c) The challenge of human autonomy. The starting point of everyone's genetic makeup is incidental, which makes us independent of other people and subject only to natural probability, and this is the key to our free will as autonomous individuals. Genetic enhancement, on the other hand, puts us under the control of others. At the same time, genetic enhancement technology also violates the informed consent principle, because the subject is still an embryo and the decision of whether or not to exert gene editing is made by its parents or some social authority. This decision may go against the attitude and choice of the embryo itself after his born and maturity. In addition, gene editing has made it easier for people to focus more on the significance of innate genes to individual success and ignore the meaning of hard work after born, and even turn them into gene-determinists. [11] In the social aspect: (a) The break of traditional fairness. The traditional view is that only God or nature have the right to produce genetic mutations, not humans. Even if human beings have the right to get their hands on genetic editing, it is more 
necessary to base it on the public interests after full discussion, rather than the monopoly interests of private or minority groups. [12] (c) The disruption of traditional views and orders. Human gene enhancement changes human physiology and psychology, which will greatly impact our traditional social concepts and orders based on our original body and mind. [13] For example, it will destroy the mobility between classes, wear down the will to struggle, reset the evaluation criteria of moral, shake the original intergenerational relationship, lead to ideas of genetic discrimination and survival of the fittest, expand social fragmentation and conflict, and so on.

From the above discussion, we can see that human embryo gene enhancement has both advantages and disadvantages for human survival and development, and the disadvantages far outweigh the benefits, so human embryo gene enhancement should be prohibited.

\section{Ethical Problems on Gene-enhancement During Human Growing Period}

Human growing period is generally recognized as the period from the ninth day after fertilization to adulthood at the age of 18. The gene enhancement performed at this stage is gene editing of somatic cells, and the difference between somatic and germline gene editing is mainly the proportion of somatic cells that will be eventually effected. Somatic gene editing usually affects only a subset of cells and is limited to a single organ (e.g., blood, liver, muscle), and does not change all somatic cells in the body as germline gene editing does. At present, there is little discussion on the ethics of gene enhancement during human growing period, and the academic circles mainly hold negative views.

On the side of supporters, the most important opinions are the three arguments mentioned above: "conducive to scientific research", "conducive to ameliorate human defects" and "conducive to the competition of our offspring". At the same time, gene enhancement during human growing period can also effectively avoid some ethical charges that are difficult to avoid for embryo gene enhancement. In the physiological aspect, the charge of "germline alteration" can be avoided. Gene-edited somatic cells cannot replace all cells throughout the body, especially the reproductive cells, and therefore cannot be inherited. Children whose somatic cells have been genetically edited only have some superficial and subtle differences from the original humans, without damaging the deep root of human germline. In most cultures, fetuses, infants children and adolescents are treated as people, not dead objects. The lives, fates and privacy involved in their gene-editing process are treated with the same respect as adults and are governed by deliberate ethical and legal norms. So as long as we make serious efforts, the dignity of people who are genetically enhanced during their growing period can be well respected and maintained.

On the side of opponents, the main idea is that genetic enhancement during human growing period changes human nature and creates new non-human attributes, thus posing a threat to the survival and development of original humans. The definition of human nature is complicated. Fukuyama believes that human nature is the common essence that behind the diversity between individuals. As for what this common essence is, Fukuyama himself is not clear and just referred to it as X. [14] But if we follow the definition of health as defined by the World Health Organization, that is, a sound and complete person, then this $\mathrm{X}$ should at least include the common essence of three aspects of physical instinct, psychological quality and social habits. The growing period is the key period for the shaping of these three common essence, and then the genetic modification carried out during this period will greatly affect the formation of human nature. (a) Although the gene enhancement during human growing period mainly changes the carrier of human physiological organs, these changes are bound to affect the physiological instincts, psychological cognition and social habits at the same time. If this is a fine tuning after the maturity of human nature, then no matter how can not get rid of the original basis of human nature. But if this is a substitution of raw materials for the construction of immature human nature, then it is a change of the original basis and will create nonhuman that will no longer bound by the original human nature. Just like the change from earthenware to porcelain. Even though the nature of an gene-enhanced individual has a lot common with the original normal human nature, the iconic event of the change of the building material during growing period has led us to conventionally identify it as a nonhuman nature, akin to a wolf child. (b) The probability of inhuman attributes due to genetic mutations and environmental differences is extremely low. Most of these attributes will be passively assimilated or actively hidden, and so to eventually integrated into the normal human culture. Nonhuman attributes that are influenced by gene editing during growing period can appear in large numbers and form an interest alliance. These attributes are often specially selected advantage attributes and will inevitably pose a survival challenge to the original human. (c) In addition, the nonhuman attributes inherent in birth are often uncontrollable and accidental products, which are inevitable appendages of human beings as a whole, and human beings as a whole should bear their advantages and disadvantages. Meanwhile, the nonhuman attributes that are influenced by gene editing during growing period are often the intentional product of the selfish interests or prejudices of a few people, but the consequences of their benefits and disadvantages would be borne by the whole of humanity, which is obviously unfair. (d) It is also important to note that the genetic enhancement during growing period is not the same as the physical, mental and social enhancement obtained through external training. The former are often seen as creating a new human being and thus lacking the identity of the human community, while the latter are often seen as tweaking the solid foundations of the existing human nature and thus still being human. At the same time, the former can be solidified at the genetic level, which leads to the high cost of change or reverse, while the latter is highly malleable 
because of the external fine-tuning obtained through training. Of course, it is impossible to draw an absolute boundary between the two, and this can only be done in a conventionalism manner similar to the ideal experiment of a smooth inclined plane, which ignores the friction.

On the side of opponents, there are some other ethical criticisms from physiological, psychological, and social perspectives. Here, just like the embryonic gene editing, the gene editing during human growing period will also face the criticism of "lack of security" and "blurred boundary" in the physiological aspect, the criticism of "autonomy challenge" in the psychological aspect, and the criticism of "break of traditional fairness" and "disruption of traditional views and orders" in the social aspect.

From the above discussion, we can see that gene enhancement during human growing period has both advantages and disadvantages for human survival and development, and the disadvantages far outweigh the benefits. Therefore, gene enhancement during human growing period should be prohibited.

\section{Ethical Problems on Gene-enhancement During Human Adulthood}

Human adulthood is generally considered to be after the age of 18 , when a person has a full and mature human nature. The genetic enhancement of adults at this stage is a kind of somatic cell editing that will not be passed down to the offspring. At the same time, it is also a independent choice based on the backbone of human nature, which will not destroy the foundation of human nature. These two major and other characteristics would largely break the ethical dilemma of genetic enhancement, but there has been little discussion about them.

On the side of supporters, the most important opinions are the three arguments mentioned above: "conducive to scientific research", "conducive to ameliorate human defects" and "conducive to the competition of our offspring". On the basis of avoiding germline change and dignity challenge in embryo, gene enhancement in adult stage can also effectively avoid some ethical charges in growing stage. In the psychological aspect, the criticism of "human nature change" and "autonomy challenge" can be avoided. As for human nature, adult genetic enhancement is grafting on the backbone of mature, fixed humanity, rather than replacing the backbone itself. This is an extension of diversity after identifying with the original human nature. The original human nature has not changed and is given a sacred status. On the basis of ensuring a sense of identity and responsibility for it, we are encouraged to explore the frontiers of human nature's plasticity. As for human autonomy, the genetic enhancement of adults is the voluntary selection on a fully informed basis, and it is also a expression of the voluntary will that is not controlled by others. Genetic enhancement in adults is more of a commodity that can be purchased at a sufficient cost after self-effort, and is an extrinsic aid. In the social aspect, the criticism of "break of traditional fairness" and "disruption of traditional views and orders" can be avoid. As for fairness, no one is God, only nature itself can play the role of God. Nature and the free market are the most fair and just. Genetic enhancement should not be monopolized by a few people, but should be based on the principles of the free market, where everyone has the opportunity to gain access to it through legitimate ways. On the basis of this fundamental principle, we can further consider macro-control based on social welfare. As for traditional views and orders, we have argued that adult genetic enhancement is an external aid to self-striving effort, which will in turn stimulates competition further. On this basis, genetic discrimination does exist, but it is as common as discrimination against disadvantaged groups in society today, and there is no essential difference. Genetic enhancement in adults, like a parent's gender change, may be a bit of a maladjustment for their children, but does not necessarily affect the underlying intergenerational relationship. This is mainly the result of adults' own hard work, like buying a mansion or living in a slum, without causing social fragmentation or social conflict. The survival of the fittest among adults is itself class mobility. Parents cannot directly pass on their genetic advantages to their children, but can only support them in the external environment. It is the children's own efforts and choices that matter, and this is no different from the current situation of social mobility.

On the side of opponents, there are left only the criticisms of "lack of security" and "blurred boundary". The criticism of "lack of security" must be accepted. Security is a prerequisite for the application of new medical technology in human experiments. But this is more of a supervision than a critic, suggesting that genetic enhancement techniques for adults must meet safety standards before they can be used. The problem of "blurred boundary" remains. But since we support adult genetic enhancement through the previous analysis, both treatment and enhancement are marketed as a medical service, so there is no need for a clear delineation at all.

From the above discussion, we can see that human adult gene enhancement is mainly beneficial to human survival and development, and even if there are some deficiencies, it can be improved continuously during development. Therefore, human adult gene enhancement should be supported.

\section{Summary and Further Extension of the Inference}

By distinguishing different stages of human development and analyzing the specific controversies surrounding gene enhancement at each stage, we conclude that gene enhancement during embryonic and growing period should be prohibited, while gene enhancement during adulthood should be supported. Other applications of human enhancement technologies, such as cosmetic surgery, stimulants, moral enhancement, brain-machine integration, and life extension, fit a similar argument. As long as it involves fundamental 
changes in germline and human nature, that is, human enhancement during embryonic and growing period, it must be prohibited. For example, cosmetic surgery for children and adolescents is strictly prohibited in many countries. But if it's a tweak of the original human germline and nature, that means human enhancement in adulthood, it should be supported. Musk's brain-machine experiments, for example, have attracted intense interest from both capital and the public. At the same time, it can better deal with the problems of "lack of security" and "blurred boundary" in physiology, the problems of "dignity challenge" and "autonomy challenge" in psychology, as well as the problems of "fairness break" and "orders disruption" in society. This is similar to the genetic enhancement argument in this paper. Human enhancement in adulthood is a fine-tuning process on the basis of maintaining the original germline and humanity, which is very similar to various existing physiological, psychological and social phenomena, and will not bring about great changes.

As for the specific advancement of adult gene enhancement and the application of other human enhancement technologies, we can adopt the strategy of "advanced drive the backward", and eventually move towards the common enhancement of all people. Everyone is precious and has a voluntary choice of whether to enhance or not. Adults with a complete physical, psychological and social structure can choose their own enhancement route according to their own will and ability. Some choose not to enhance, some choose to enhance conservatively, and some choose to enhance radically, thus forming different echelon levels. But no matter in which level of the members are a member of the human family, have contributed to the development of human civilization. This force may be positive, negative or zero, and its due benefits or punishments should be fairly distributed in proportion to its contribution to human civilization. The fixed buyout salary that we used to have for workers are not consistent with this dynamic benefits. Only the shareholding system of the whole people is the real fairness and justice. The very nature of the shareholding system also dictates that no one should be left behind in the enterprise of human enhancement. [15]

We should give priority to ensuring the minimum survival and development of the unenhanced class that is the common source of mankind. They are the carrier of all human enhancement technologies and the common root of enhanced post-human beings, which can bring together the scattered new human beings and glue them together into a common human civilization. This unenhanced class is less competitive than the enhanced post-human class and, according to the previous discussion of the universal shareholding system, they are bound to enjoy the lowest proportion of welfare. If this minimum proportion of distributed welfare can not maintain the minimum survival needs of each member within it, then it will inevitably damage the basic roots of the human community. This will cause human enhancement to become water without source. Such minimum welfare should guarantee the basic survival of the mainstream normal individuals in the unenhanced stratum. It's like a low-grade version of the Great Harmony society or the Communist society. Humans who choose enhancement obviously cannot do like this. Differences in the direction, level and effect of enhancement will lead to differences in individual endowments. Differences in individual endowments will lead to differences in the division of labor that may be engaged in society. The difference of social division of labor will further lead to the difference of social class. The greater the difference, the farther away from the great unity of communism. This is true in the early days after a technological breakthrough. In incremental competition, some individuals have a first-mover advantage in their ideas, abilities, and resources, which will further widen the gap with others in subsequent competition. But as technology reaches a plateau or even a bottleneck, that will change. In inventory competition, some people's first-mover advantage will be gradually leveled, and the gap between people will gradually narrow. Whether it is incremental competition or stock competition, those who choose to enhance must compete. Competition will produce the first person and the last person, but here is not the survival of the fittest, but the advanced intensifies the competition and the backward slowly catch up. Of course, laggards can also choose to shed all their enhancement applications and move to the unenhanced class. People in the unenhanced class are very similar in endowment, division of labor and class, and enjoy similar welfare, and this welfare will gradually increase with the development of the whole human society.

\section{Acknowledgements}

This thesis is supported by the Social Science Foundation of Guizhou Province, NO: 19GZYB61.

\section{References}

[1] Kate Williams; Martin H Johnson. Adapting the 14-day rule for embryo research to encompass evolving technologies. [J]. Reproductive biomedicine \& society online. 2020: 1-9.

[2] Savulescu, Julian. An ethical pathway for gene editing. [J]. Bioethics. 2019, Vol. 33 (No. 2): 221-222.

[3] Paul Knoepfler. GMO Sapiens: The Life-Changing Science of Designer Babies [M]. Singapore: World Scientific Publishing, 2015.

[4] Gyngell C, Douglas T, Savulescu J. The Ethics of Germline Gene Editing [J]. Journal of Applied Philosophy, 2016 (4): $1-16$.

[5] Hauskeller, Michael. Better Humans? Understanding the Enhancement Project [M]. New York: Routledge. 2014.

[6] Isasi R, Kleiderman E, Knoppers BM. Genetic technology regulation. Editing policy to fit the genome? [J]. Science, 2016 (351): 337-339.

[7] David Baltimore, Paul Berg, Michael Botchan. A prudent path forward for genomic engineering and germ-line gene modification [J]. Science, 2015 (348): 36-38. 
[8] Juengst E. T. What Does Enhancement Mean? [C]. in E. Parens (ed.). Enhancing Human Traits: Ethical and Social Implications. Washington DC: Georgetown University Press, 1998: 29—47.

[9] Sandel M, The case against perfection [M]. Cambridge, M. A: Harvard University Press, 2009: 85-90.

[10] Ronald Dworkin. Playing God: Genes, Clones, and Luck [C]. in Ronald Dworkin (ed.). Sovereign Virtue: The Theory and Practice of Equality. Cambridge: Harvard University Press, 2000: 439.

[11] Kelly E, et al. Human Germline Genome Editing [J]. American Journal of Human Genetics, 2017 (101): 167-176.

[12] Benjamin Capps, ed al. Falling giants and the rise of gene editing: ethics, private interests and the public good. [J]. Hum Genomics, 2017 (1): 20.
[13] The Center for Genetics and Society. Public interest group calls for strengthening global policies against human germline modification' [online]. https://www.geneticsandsociety.org/press-statement/public-int erest-group-calls-strengthening-global-policies-against-human -germline?id=8528.

[14] Fukuyama F. Our Posthuman Future: Consequences of the Biotechnology Revolution [M]. New York NY: Farrar, Straus, and Giroux. 2002: 14-152.

[15] Jingtao Jiang. A Naturalistic Approach of Human Enhancement Ethics. Science, Technology \& Public Policy. Vol. 4, No. 2, 2020, pp. 54-59. 